\title{
Finite Element Analysis of Abdominal Aortic Aneurysms: Predicted Rupture Risk Correlates With Aortic Wall Histology in Individual Patients
}

\author{
Philipp Erhart, $\mathrm{MD}^{1}$; Caspar Grond-Ginsbach, $\mathrm{PhD}^{2}$; Maani Hakimi, $\mathrm{MD}^{1}$; Felix Lasitschka, $\mathrm{MD}^{3}$; \\ Susanne Dihlmann, $\mathrm{PhD}^{1}$; Dittmar Böckler, $\mathrm{MD}, \mathrm{PhD}^{1}$; and Alexander Hyhlik-Dürr, $\mathrm{MD}, \mathrm{PhD}^{1}$ \\ Departments of ${ }^{1}$ Vascular and Endovascular Surgery, ${ }^{2}$ Neurology, and ${ }^{3}$ Pathology, \\ Ruprecht-Karls University Heidelberg, Germany.
}

\begin{abstract}
Purpose: To evaluate a finite element analysis (FEA) model as a predictive tool for abdominal aortic aneurysm (AAA) rupture risk assessment.

Methods: FEA of asymptomatic infrarenal AAAs in 15 men (mean age 72 years) was performed preoperatively using semiautomatic finite element analysis software (A4clinics) to calculate peak wall stress (PWS) and regions of highest and lowest rupture risk index (RRI). The areas of high and low RRI identified on the preoperative FEA were sampled during open surgery; aortic wall specimens were prepared for histological analysis. A semiquantitative score compared the histological findings from high and low rupture risk samples.

Results: Significant correlation was found between histological AAA wall integrity and RRI in individual patients. AAA wall regions with highest RRI showed advanced histological disintegrity compared to regions with lower RRI within the same AAA: mean smooth muscle cells: 0.43 vs. 1.21 , respectively ( $p=0.031$ ); elastic fibers: 0.57 vs. 1.29 , respectively ( $p=0.008$ ); cholesterol plaque: 2.60 vs. 2.20 , respectively ( $p=0.034$ ); and calcified plaque: 2.27 vs. 1.40 , respectively $(\mathrm{p}=0.017)$. The amount of calcified plaque was significantly correlated with PWS $(r=0.528, p=0.043)$ by univariate regression analysis. However, there was no correlation between PWS or RRI and the histological findings between patients.

Conclusion: These preliminary results show that high rupture risk regions estimated by FEA contain increased histopathological degeneration compared to low rupture risk samples within the same AAA. Until now, the role of FEA in predicting individual AAA rupture risk has not been established as a validated diagnostic tool. However, these data provide promising results for FEA model verification.
\end{abstract}

Key words: aorta, abdominal aortic aneurysm, finite element analysis, histology, rupture risk, model verification, rupture risk index

Ruptured abdominal aortic aneurysms (AAAs) are associated with $70 \%$ to $90 \%$ mortality and represent a relevant cause of death for men in Western societies. ${ }^{1}$ The ability to predict an individual patient's rupture risk and perform elective surgical repair of high-risk AAAs are important clinical issues; however, prediction of AAA rupture risk remains uncertain. While maximal AAA diameter $>5.5 \mathrm{~cm}$ or rapid AAA diameter expansion are determining parame-

The authors declare no association with any individual, company, or organization having a vested interest in the subject matter/products mentioned in this article.

Corresponding author: Alexander Hyhlik-Dürr, MD, PhD, Department of Vascular and Endovascular Surgery, Ruprecht Karls University Heidelberg, Im Neuenheimer Feld 110, 69120 Heidelberg, Germany. E-mail: alexander.duerr@med. uni.heidelberg.de 
ters for surgical repair, ${ }^{2}$ several studies have shown that rupture occurs in some AAAs $<5.5$ $\mathrm{cm}$ in diameter ${ }^{3}$ and never in others measuring $>5.5 \mathrm{~cm}^{4}$

Finite element analysis (FEA) is a computational method of estimating biomechanical parameters based on patient-specific determinants such as blood pressure and AAA geometry reconstructed from computed tomographic angiography (CTA) data. The FEA model used in this study incorporates several

See commentary page 565

AAA rupture risk factors, i.e., AAA morphology, ${ }^{5}$ gender ${ }^{6-8}$ arterial hypertension, ${ }^{9}$ smoking history, ${ }^{10}$ familial AAA predisposition, ${ }^{11}$ and the amount of intraluminal thrombus. ${ }^{12}$

After reconstruction of AAA geometry, biomechanical calculations are based on the finite element method, i.e., segmentation of the 3-dimensional (3D) AAA contour model into multiple hexahedral volume meshes. Biomechanical parameters are subsequently computed and illustrated within the AAA contour model. The peak wall stress (PWS) depends on blood pressure and AAA morphology and represents the greatest pressure value transmitted on a certain region of the AAA wall. The rupture risk index (RRI; wall stress divided by strength of the AAA wall), additionally incorporates the wall weakening effects of intraluminal thrombus, which has been described by isotropic models before. ${ }^{13}$ The maximal value of RRI within an AAA is referred to as the peak wall rupture risk (PWRR). Early FEA results revealed higher PWS and PWRR in ruptured AAAs compared to asymptomatic AAAs, ${ }^{8,14,15}$ and several independent studies have demonstrated that PWS and PWRR are more reliable rupture risk indicators than AAA diameter alone. ${ }^{14,16,17}$ However, individual FEA-based rupture risk estimation has not been verified with histological findings from the AAA wall. This study quantifies and compares histological degeneration from AAA wall regions identified as having high or low RRI based on FEA.

\section{METHODS}

\section{Study Patients and Imaging Acquisition}

In a single-center study approved by the university's medical ethics committee, 37 patients with asymptomatic infrarenal AAAs $>50 \mathrm{~mm}$ in diameter scheduled for elective surgical repair between June 2010 until June 2013 were enrolled after giving written informed consent.

Preoperative CTAs were generated with a 64-slice CT scanner (Somatom Definition; Siemens, Munich, Germany) using standard parameters (in plane resolution $0.33 \mathrm{~mm}$, slice thickness $0.7-3.3 \mathrm{~mm}$ ) and intravenous application of iodinated contrast (Ultravist 370; Berlex Laboratories, Wayne, NJ, USA). Brachial systolic and diastolic blood pressure measurements were determined prior to and after CTA. The average blood pressure values, which were within the $120-150 \mathrm{mmHg}$ systolic range, were used for subsequent finite element calculation.

\section{FEA Rupture Risk Assessment}

Using the A4clinics finite element analysis software (VASCOPS GmbH, Graz, Austria), which has been shown to have high inter- and intrapersonal reproducibility for deriving FEA parameters, ${ }^{18,19}$ a single investigator reconstructed a 3-dimensional contour model of each subject's AAA from the preoperative CTA data contained in a DICOM (Digital Imaging and Communication in Medicine) file. Image segmentation was performed using methods reported previously. ${ }^{19}$

FEA was performed from below the renal arteries to above the aortic bifurcation ${ }^{13}$ to calculate the following parameters: PWS (in kilopascal, $\mathrm{kPa}$ ), PWRR, maximal AAA diameter (in $\mathrm{mm}$ ), total AAA lumen volume (in $\mathrm{cm}^{3}$ ), intraluminal thrombus volume (in $\mathrm{cm}^{3}$ ), and rupture risk equivalent diameter (RRED; in $\mathrm{mm}$ ) for each patient (Table 2). The RRED is a virtual value expressing the maximum diameter of an "average AAA" (based on epidemiologic and biomechanical data) with the same risk of rupture (i.e., the same PWRR) ${ }^{8,20}$ Since PWRR may discriminate rupture risk slightly better than PWS $^{13}$ for each 3D AAA contour 
TABLE 1

Baseline Patient Characteristics and Comorbidities

\begin{tabular}{ll}
\hline Age, y & $72.2 \pm 5.7[60-84]$ \\
Men & $15(100 \%)$ \\
Arterial hypertension & $14(93.3 \%)$ \\
Smoking history & $9(60.0 \%)$ \\
Coronary heart disease & $7(46.7 \%)$ \\
Dyslipidemia & $5(33.3 \%)$ \\
COPD & $3(20.0 \%)$ \\
Renal insufficiency & $3(20.0 \%)$ \\
Peripheral artery disease & $2(13.2 \%)$ \\
Diabetes mellitus & $1(6.7 \%)$
\end{tabular}

Continuous data are presented as the means \pm standard deviation [range]; categorical data are given as the counts (percentage).

COPD: chronic obstructive pulmonary disease.

model a RRI color scaling was performed mapping regions of lowest and highest $R R I$ regions for each $A A A$. No threshold values for these RRI were respected, i.e., tissue extraction during surgery from the highest RRI (i.e., region of $P W R R$ ) and lowest $R R I$ in each patient was based on individual RRI color scaling alone. Tissues were collected and processed according to ethical guidelines and the standard operating protocols of our department's vascular biobank.

\section{Tissue Sampling and Histological Analysis}

At open surgical repair, AAA wall samples measuring $10 \times 10 \mathrm{~mm}^{2}$ were extracted during aortic cross-clamping and fixed in $3.7 \%$ formaldehyde for histological analysis (Fig. 1). After ethanol dehydration and embedding in paraffin, each sample was cut into 2 to $4-\mu \mathrm{m}$ sections. Following deparaffination and rehydration, microscopic sections were contrasted by hematoxylin \& eosin staining using standard laboratory protocols.

Microscopic semiquantitative analysis was performed by two observers (vascular surgeon and biologist) who independently graded the amounts of smooth muscle cells (SMCs), elastic fibers, atherosclerotic plaque (including cholesterol and calcified plaques), fibrosis (amount of collagen deposition), inflammation (vascular-associated lymphoid
TABLE 2

Preoperative FEA Parameters

\begin{tabular}{ll}
\hline Peak wall stress, $\mathrm{kPa}$ & $209 \pm 40[138-293]$ \\
Peak wall rupture risk & $0.48 \pm 0.13[0.27-0.79]$ \\
$\begin{array}{l}\text { Maximum AAA } \\
\text { diameter, mm }\end{array}$ & $62.0 \pm 9.5[52.2-85.2]$ \\
$\begin{array}{l}\text { Total AAA lumen } \\
\quad \text { volume, } \mathrm{cm}^{3}\end{array}$ & $85.7 \pm 27.6[40.5-141.0]$ \\
$\begin{array}{l}\text { Volume of intraluminal } \\
\text { thrombus, } \mathrm{cm}^{3}\end{array}$ & $99.4 \pm 80.6[15.6-326.6]$ \\
$\begin{array}{l}\text { Rupture risk equivalent } \\
\quad \text { diameter, mm }\end{array}$ & $57.8 \pm 11.6[35.2-80.7]$
\end{tabular}

Data are presented as the means \pm standard deviation [range].

FEA: finite element analysis, $\mathrm{kPa}$ : kilopascal, $\mathrm{AAA}$ : abdominal aortic aneurysm.

tissue $\left.^{21}\right)$, and vasa vasorum and nervi vasorum (Fig. 2) using a predefined descriptive histology score (0: absence, 1: strongly reduced amount, 2: moderately reduced amount, 3: normal or increased amount), which was adapted from Rijbroek et al. ${ }^{17}$ Samples found to have incomplete wall extraction or severe atherosclerotic wall degeneration were excluded from the correlation analysis.

\section{Statistical Analysis}

Low and high risk samples were compared using the Wilcoxon paired test, while histopathological findings were compared with FEA parameters using the Spearman correlation test. A univariate regression analysis was used to investigate any correlation between patient baseline demographics or comorbidities and the histological results. $\mathrm{P}<0.05$ was considered the threshold of significance. The analyses were performed using the SPSS statistics software package (version 19.0; IBM Corporation, Somers, NY, USA).

\section{RESULTS}

Aneurysm wall tissue was extracted from all 37 patients, though in some cases, tissue extraction from the posterior portion of the AAA wall could not be performed if iatrogenic bleeding from the inferior vena cava was to be avoided. Among the 37 study patients, spec- 


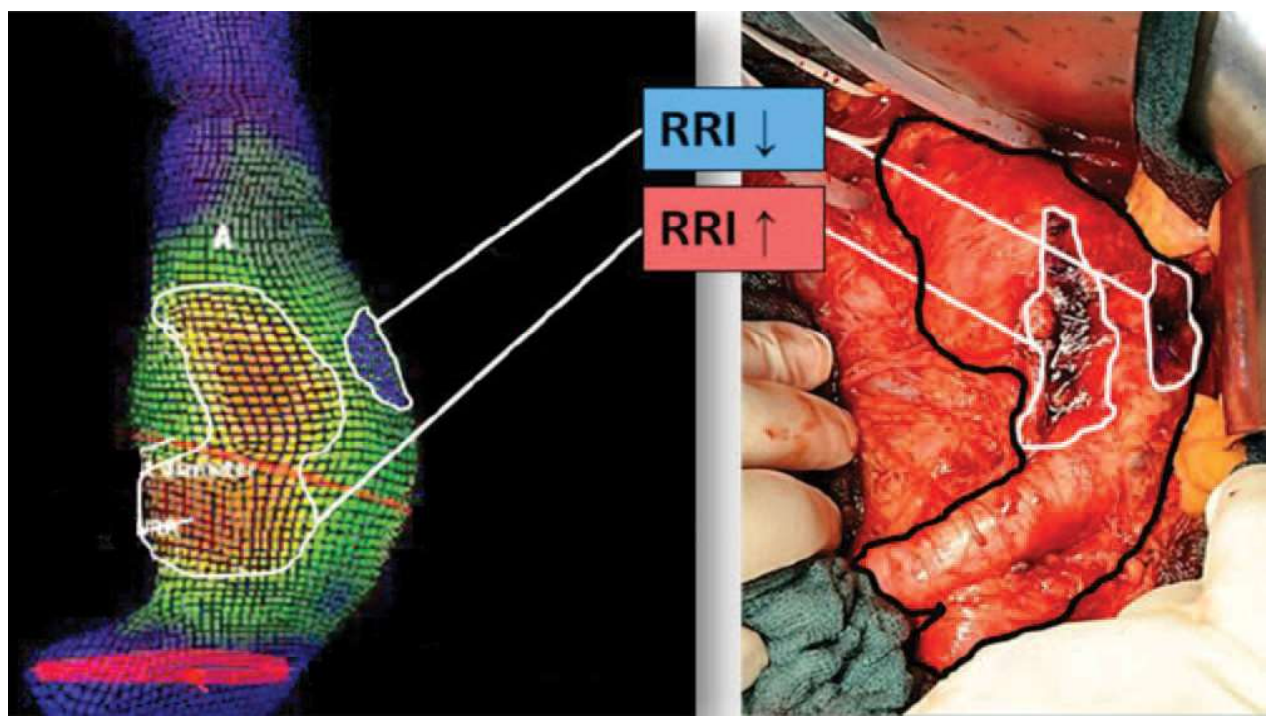

Figure $1 \diamond$ Based on preoperative FEA, regions of the AAA wall with estimated lowest $(\downarrow)$ and highest (PWRR) $(\uparrow)$ rupture risk could be identified using RRI color scaling. During open surgical intervention, aortic wall samples were extracted from these regions.

imens from 22 patients were not suitable for the correlation analysis, leaving 15 male patients (mean age 72 years, range $60-84$ ) in the study cohort. Their characteristics are shown in Table 1.

Among the eligible patients, 15 samples from high RRI regions were extracted from the anterior $(46.7 \%)$, left lateral $(26.7 \%)$, right lateral $(20.0 \%)$, and posterior $(6.6 \%)$ aspects of the AAA, while 15 low RRI samples were taken from the anterior $(66.7 \%)$, left lateral $(13.3 \%)$, right lateral $(6.7 \%)$, and posterior $(13.3 \%)$ part of the AAA wall.

Histological comparison of the AAA wall samples from the highest and lowest RRI regions for each patient found significantly more SMCs $(p=0.031)$ and elastic fibers $(p=0.008)$ in samples with low RRI, suggesting increased local wall integrity (Table 3 ). The amount of atherosclerotic plaque, including cholesterol and calcified plaque, was significantly increased in samples at high RRI $(p=0.034$ and $p=0.017$, respectively). Cholesterol plaque was found in variable amounts in all AAA specimens, except one low RRI sample. Out of 30 AAA samples, six at low risk and one at high RRI were devoid of calcified plaque. Hence, based on finite element calculation, histological differences within the same AAA were registered for parameters belonging to the tunica intima and tunica media (SMCs, elastic fibers, and atherosclerotic plaque).

In our study, the grade of inflammation and the amount of vasa vasorum and nervi vasorum did not differ significantly (tunica adventitia), whereas high RRI samples tended to have pronounced fibrosis ( $p=0.085$ ). Evidence of inflammatory cell infiltrates could be found in every AAA sample. Tertiary lymphoid tissue aggregates, indicators of intense chronic inflammation, were observed within the tunica adventitia (Fig. 3 ) in six AAA samples (low/high RRI ratio $=1 / 5$ ). Lymphocytic cell infiltrates were present predominantly along the border of the tunica media and tunica adventitia. If increased fibrosis existed, inflammatory cell infiltrates assembled in regions of original non-fibrous tunica adventitia. Fibrosis could be found especially between the tunica media and tunica adventitia (Fig. 3).

PWS was associated with greater amounts of calcified plaque, i.e., AAAs with higher PWS from preoperative FEA showed increased amounts of calcified plaque compared to AAA with lower PWS ( $p=0.043$, bslope 0.528 by univariate regression analysis). No further correlations could be found be- 

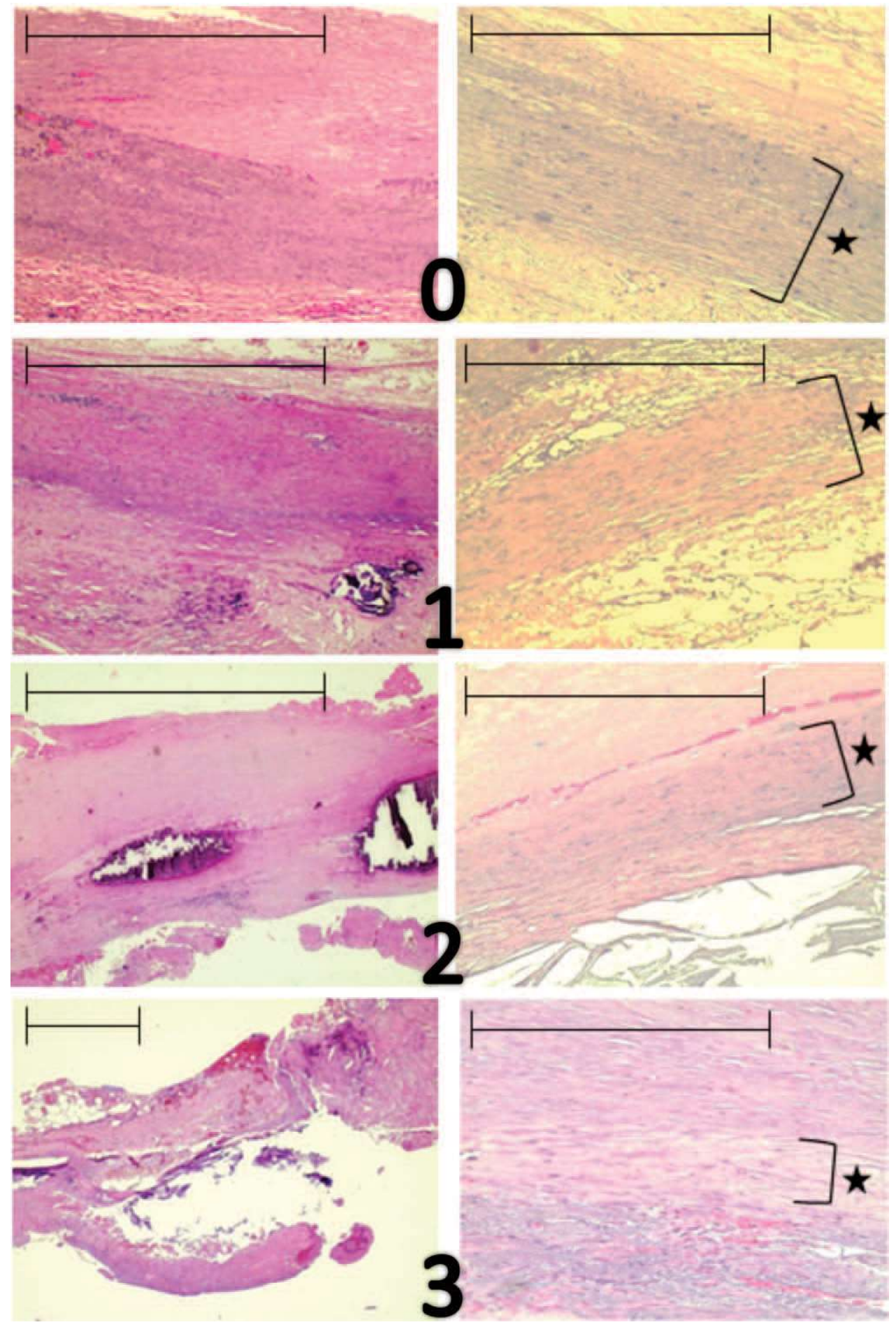

Figure 2 An illustration of the semiquantitative histology grading score used to determine the amount of calcified plaque, cholesterol plaque, inflammatory cell infiltrate, vasa vasorum, nervi vasorum, smooth muscle cells, and elastic fibers. The quantity of calcified plaque increased from grade 0 to 3 (left column), whereas elastic fiber content and total media thickness decreased from grade 0 to 3 (see asterisk right column). Bar equals $1 \mathrm{~mm}$. 
TABLE 3

Intrapatient Analysis of AAA Wall Histology

\begin{tabular}{lccr}
\hline Histology Parameter & Low-Risk Sample & High-Risk Sample & $\mathrm{p}$ \\
\hline Smooth muscle cell* $^{*}$ & $1.21[0-2]$ & $0.43[0-1]$ & 0.031 \\
Elastic fiber* $^{*}$ & $1.29[0-3]$ & $0.57[0-1]$ & 0.008 \\
Soft plaque* $^{*}$ & $2.20[0-3]$ & $2.60[1-3]$ & 0.034 \\
Hard plaque $^{*}$ & $1.40[0-3]$ & $2.27[0-3]$ & 0.017 \\
Fibrosis $^{\dagger}$ & $1.53[0-3]$ & $2.21[0-3]$ & 0.085 \\
Inflammation $^{\dagger}$ & $1.73[1-3]$ & $2.07[0-3]$ & 0.361 \\
Vasa vasorum $^{\dagger}$ & $1.87[1-3]$ & $1.31[0-3]$ & 0.191 \\
Nervi vasorum $^{\dagger}$ & $1.73[0-3]$ & $1.13[0-3]$ & 0.285
\end{tabular}

Data are presented as the mean of the histology score [range].

AAA: abdominal aortic aneurysm.

* Tunica intima/media.

$\dagger$ Tunica adventitia.

tween histopathological findings, patient characteristics, and FEA parameters (interpatient univariate regression analysis). Therefore, AAAs with higher PWRR or PWS were not associated with advanced histological degeneration compared to AAAs with lower

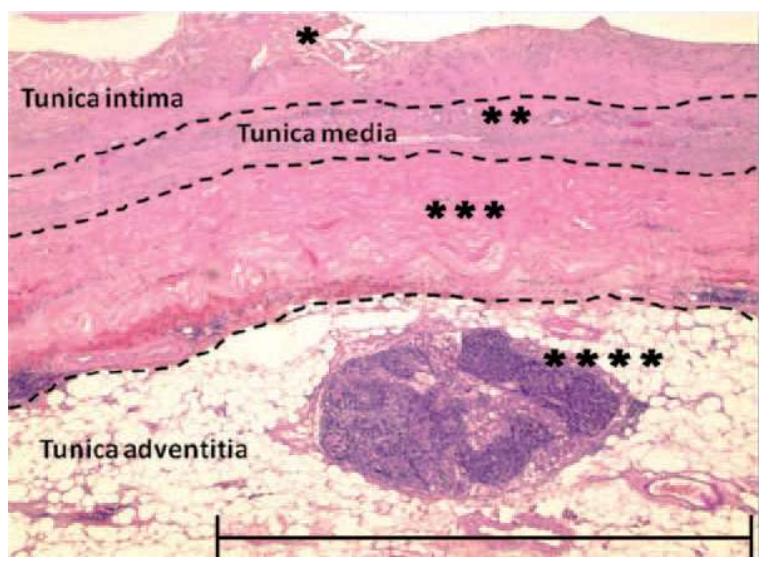

Figure $3 \bullet$ Representative section of AAA wall histology (H\&E staining). Atherosclerotic lesions, here cholesterol plaque $\left({ }^{*}\right)$, were located predominantly within the tunica intima and tunica media. The thickness of the tunica media was frequently decreased due to loss of smooth muscle cells and elastic fibers $\left({ }^{*}\right)$. Fibrosis was prominent as thick linear shaped collagen deposition on the border of the tunica media and tunica adventitia $(* * *)$. If fibrosis was present, inflammatory cell infiltrates were located mainly within primary regions of the tunica adventitia. Vascular associated lymphoid tissues were restricted to the tunica adventitia $(* * *)$. Bar equals $1 \mathrm{~mm}$.
PWRR or PWS. However, within an individual AAA, histological wall integrity was reduced in high RRI samples compared to the low RRI specimens. According to FEA, the mean value of PWRR was $0.48 \pm 0.13$ (range $0.27-0.79$ ) and the mean value of RRED was $57.8 \pm 11.6$ (range 35.2-80.7). Seven of the 15 patients undergoing surgical intervention had RRED values $<50 \mathrm{~mm}$.

\section{DISCUSSION}

This study showed that FEA rupture risk profiling (PWRR) correlates with histological degeneration of the individual AAA wall. Compared to samples with low RRI, pronounced histological degeneration was found in samples with high RRI (intrapersonal analysis). The results from histological analysis, however, did not correlate with RRI values between patients (interpersonal analysis). Tissue degeneration was detected in all layers of the AAA wall. Compared to samples with low RRI, samples with the highest RRI, i.e., the region of PWRR, had pronounced histological degeneration (SMCs, elastic fibers, atherosclerotic plaque), particularly within the tunica intima and tunica media.

The amount of inflammation ${ }^{17}$ or atherosclerosis $^{22}$ has been semiquantitatively characterized using 4-grade scoring systems by others, but we used a scoring system that considered more than one histological parameter for AAA wall degeneration. Weaken- 
ing of the AAA wall is estimated to result from degenerative processes leading to a lower amount of SMCs and elastic fibers. ${ }^{23,24}$ Whether biomechanical characteristics, i.e., pressure and wall stress, induce histopathological changes in the AAA wall has to be investigated in further studies.

Intraluminal thrombus (ILT) could not be studied in our histological analysis since portions of ILT were apparently lost during histological processing. However, Kazi et al. ${ }^{25}$ have already reported that AAA regions with the highest amount of intraluminal thrombus based on CTA data had less elastin and fewer SMCs, but more inflammatory cell infiltrates. Other authors ${ }^{26}$ assume that it is not the amount of ILT that is different between ruptured and non-ruptured AAAs but rather the eccentric ILT distribution and local thickness discrepancies within AAAs that influence wall integrity. ${ }^{27}$ Our study could not address the impact of ILT on biomechanical parameters or AAA wall degeneration.

Histological findings within the tunica adventitia (fibrosis, inflammation, vasa vasorum, and nervi vasorum) were not correlated with FEA findings. Inflammation was noticed predominantly within the tunica adventitia, supporting the "outside-in" hypothesis of AAA wall inflammation reported by Maiellaro et al. ${ }^{28}$ in 2007 . Severe fibrosis was seen in connection with advanced AAA wall inflammation, corresponding to Rijbroek's histological observations (grade 3, histological inflammation scale of aneurysm). ${ }^{17}$ Aneurysm wall fibrosis, in the form of collagen deposition, was prominent along the border of the tunica media and tunica adventitia. These results support observations of Maiellaro et al., ${ }^{28}$ suggesting that residual adventitial fibroblasts may undergo a phenotypical switch into collagen-secreting myofibroblasts.

Vascular-associated lymphoid tissue consisting of immunocompetent cells, such as B-, $\mathrm{T}-$, and dendritic cells and macrophages, may represent chronic inflammation within the AAA wall and are suspected of inducing AAA progression. ${ }^{21,29}$ Gene expression analysis of these lymphoid aggregates using realtime polymerase chain reaction revealed significantly increased amounts of AIM2 (absent in melanoma 2) and mature caspase-5 inflammasome components in high RRI samples compared to low RRI samples. ${ }^{30}$ Triggers for chronic inflammatory reaction in AAA pathogenesis remain uncertain, although inflammatory cell distribution in our study assumes adventitial origin.

On average, PWRR was $<0.5$ for the asymptomatic AAAs in this study. In 7 of the 15 patients, the associated RRED was $<50$ $\mathrm{mm}$ even though all patients showed external AAA diameters $>50 \mathrm{~mm}$, supporting the decision for surgical repair. Which FEA parameter (e.g., PWRR, PWS, or RRED) most precisely predicts AAA rupture risk remains to be evaluated.

A correlation between histopathological findings, patient characteristics, and FEA parameters could be found only for PWS and calcified plaque amount. Li et al. ${ }^{31}$ suggested that the presence of AAA wall calcification decreases AAA wall stability and hence increases local PWS, which our results can confirm. The FEA model of this study did not incorporate the amount of calcified plaque for biomechanical rupture risk computation. Whether this parameter has influence on finite element computation and improves model accuracy remains to be proven in further trials.

No patient-specific characteristic correlated with histopathological findings in our study. Similar results were observed by Hellenthal et al., ${ }^{32}$ who reported that medial thickness or inflammatory cell count of ruptured vs. nonruptured AAA wall samples was not related to maximal AAA diameter or the clinical constitution of the patients. It is likely that multiple heterogeneous parameters (e.g., genetic variables) may contribute to AAA rupture.

\section{Limitations}

This preliminary study was limited by a high number of ineligible samples due to massive histological degeneration and by the inability to extract tissue from some posterior AAA walls. In addition, no threshold values for "lowest" and "highest (i.e., individual PWRR)" RRIs were used for subsequent tissue extraction; hence, AAA wall samples might not be comparable between patients. 


\section{Conclusion}

This FEA model provides objective biomechanical parameters that may complement clinical decision making regarding AAAs. Nevertheless, evaluation of patient-specific rupture risk based on FEA alone is still undetermined. This initial attempt to correlate results from FEA with histological findings of the AAA wall shows promising results within patients. FEA model accuracy still has to be validated but may provide a more individualized rupture risk prediction in AAA patients.

Acknowledgments: The authors would like to thank Anja Spieler and Carolin Kerber for their technical assistance in tissue processing.

\section{REFERENCES}

1. Gillum RF. Epidemiology of aortic aneurysm in the United States. J Clin Epidemiol. 1995;48: 1289-1298.

2. Chaikof EL, Brewster DC, Dalman RL, et al. The care of patients with an abdominal aortic aneurysm: The Society for Vascular Surgery practice guidelines. J Vasc Surg. 2009;50(8S): 1S-49S.

3. Nicholls SC, Gardner JB, Meissner MH, et al. Rupture in small abdominal aortic aneurysms. J Vasc Surg. 1998;28:884-888.

4. Darling RC, Messina CR, Brewster DC, et al. Autopsy study of unoperated abdominal aortic aneurysms. The case for early resection. Circulation. 1977;56:II161-164.

5. Vorp DA, Raghavan ML, Webster MW. Mechanical wall stress in abdominal aortic aneurysm: influence of diameter and asymmetry. $J$ Vasc Surg. 1998;27:632-639.

6. Brown PM, Zelt DT, Sobolev B. The risk of rupture in untreated aneurysms: the impact of size, gender, and expansion rate. J Vasc Surg. 2003;37:280-284.

7. Derubertis BG, Trocciola SM, Ryer EJ, et al. Abdominal aortic aneurysm in women: prevalence, risk factors, and implications for screening. J Vasc Surg. 2007;46:630-635.

8. Gasser TC, Nchimi A, Swedenborg J, et al. A novel strategy to translate the biomechanical rupture risk of abdominal aortic aneurysms to their equivalent diameter risk: method and retrospective validation. Eur J Vasc Endovasc Surg. 2014;47:288-295.

9. Brown LC, Powell JT. Risk factors for aneurysm rupture in patients kept under ultrasound surveillance. UK Small Aneurysm Trial Participants. Ann Surg. 1999;230:289-296.

10. Powell JT, Worrell P, MacSweeney ST, et al. Smoking as a risk factor for abdominal aortic aneurysm. Ann NY Acad Sci. 1996;800:246248.

11. Larsson E, Granath F, Swedenborg J, et al. A population-based case-control study of the familial risk of abdominal aortic aneurysm. $J$ Vasc Surg. 2009;49:47-50.

12. Hans SS, Jareunpoon $O$, Balasubramaniam M, et al. Size and location of thrombus in intact and ruptured abdominal aortic aneurysms. $J$ Vasc Surg. 2005;41:584-588.

13. Gasser TC, Auer M, Labruto F, et al. Biomechanical rupture risk assessment of abdominal aortic aneurysms: model complexity versus predictability of finite element simulations. Eur J Vasc Endovasc Surg. 2010;40:176-185.

14. Maier A, Gee MW, Reeps C, et al. A comparison of diameter, wall stress, and rupture potential index for abdominal aortic aneurysm rupture risk prediction. Ann Biomed Eng. 2010;38: 3124-3134.

15. Venkatasubramaniam AK, Fagan MJ, Mehta T, et al. A comparative study of aortic wall stress using finite element analysis for ruptured and non-ruptured abdominal aortic aneurysms. Eur J Vasc Endovasc Surg. 2004;28:168-176.

16. Teutelink A, Cancrinus $E$, van de Heuvel D, et al. Preliminary intraobserver and interobserver variability in wall stress and rupture risk assessment of abdominal aortic aneurysms using a semiautomatic finite element model. $J$ Vasc Surg. 2012;55:326-330.

17. Rijbroek A, Moll FL, von Dijk HA, et al. Inflammation of the abdominal aortic aneurysm wall. Eur J Vasc Surg. 1994;8:41-46.

18. Hyhlik-Dürr $A$, Krieger $T$, Geisbüsch $P$, et al. Reproducibility of deriving parameters of AAA rupture risk from patient-specific 3D finite element models. J Endovasc Ther. 2011;18: 289-298.

19. Auer M, Gasser TC. Reconstruction and finite element mesh generation of abdominal aortic aneurysms from computerized tomography angiography data with minimal user interactions. IEEE Trans Med Imaging. 2010;29:10221028.

20. Roy J, Swedenborg J, Sakalihasan N, et al. Biomechanical rupture risk assessment of AAA made easier for clinicians. J Vasc Surg. 2012; 55:29S-30S

21. Bobryshev YV, Lord RS. Vascular-associated lymphoid tissue (VALT) involvement in aortic aneurysm. Atherosclerosis. 2001;154:15-21. 
22. Koole D, Hurks R, Schoneveld A, et al. Osteoprotegerin is associated with aneurysm diameter and proteolysis in abdominal aortic aneurysm disease. Arterioscler Thromb Vasc Biol. 2012;32:1497-1504.

23. Hellenthal FA, Buurman WA, Wodzig WK, et al. Biomarkers of AAA progression. Part 1: extracellular matrix degeneration. Nat Rev Cardiol. 2009;6:464-474.

24. Michel JB, Martin-Ventura JL, Egido J, et al. Novel aspects of the pathogenesis of aneurysms of the abdominal aorta in humans. Cardiovasc Res. 2011;90:18-27.

25. Kazi M, Thyberg J, Religa $P$, et al. Influence of intraluminal thrombus on structural and cellular composition of abdominal aortic aneurysm wall. J Vasc Surg. 2003;38:1283-1292.

26. Golledge J, lyer V, Jenkins J, et al. Thrombus volume is similar in patients with ruptured and intact abdominal aortic aneurysms. J Vasc Surg. 2014;59:315-320.

27. Koole D, Zandvoort HJ, Schoneveld A, et al. Intraluminal abdominal aortic aneurysm thrombus is associated with disruption of wall integrity. J Vasc Surg. 2013;57:77-83.

28. Maiellaro K, Taylor WR. The role of the adventitia in vascular inflammation. Cardiovasc Res. 2007;75:640-648.

29. Ocana E, Bohorquez JC, Perez-Requena J, et al. Characterisation of $\mathrm{T}$ and $\mathrm{B}$ lymphocytes infiltrating abdominal aortic aneurysms. Atherosclerosis. 2003;170:39-48.

30. Dihlmann S, Erhart P, Mehrabi A, et al. Increased expression and activation of absent in melanoma 2 inflammasome components in lymphocytic infiltrates of abdominal aortic aneurysms. Mol Med. 2014 Mar 5. [Epub ahead of print].

31. Li ZY, U-King-Im J, Tang TY, et al. Impact of calcification and intraluminal thrombus on the computed wall stresses of abdominal aortic aneurysm. J Vasc Surg. 2008;47:928-935.

32. Hellenthal FA, Geenen IL, Teijink JA, et al. Histological features of human abdominal aortic aneurysm are not related to clinical characteristics. Cardiovasc Pathol. 2009;18: 286-293. 